\title{
The association between the extent of lipidic burden and delta- fractional flow reserve: analysis from coronary physiological and near-infrared spectroscopic measures
}

\author{
Kota Murai ${ }^{1,2}$, Yu Kataoka ${ }^{1,2}$, Yuriko Nakaoku ${ }^{3}$, Kunihiro Nishimura ${ }^{3}$, Satoshi Kitahara ${ }^{1,2}$, \\ Takamasa Iwai ${ }^{1}$, Hayato Nakamura ${ }^{4}$, Hayato Hosoda ${ }^{1}$, Atsushi Hirayama ${ }^{1}$, Hideo Matama ${ }^{1,2}$, Takahito Doi ${ }^{1}$, \\ Takahiro Nakashima ${ }^{1}$, Satoshi Honda ${ }^{1}$, Masashi Fujino ${ }^{1}$, Kazuhiro Nakao ${ }^{1}$, Shuichi Yoneda ${ }^{1}$, \\ Kensaku Nishihira ${ }^{5}$, Tomoaki Kanaya ${ }^{6}$, Fumiyuki Otsuka ${ }^{1}$, Yasuhide Asaumi ${ }^{1}$, Kenichi Tsujita $^{7}$, \\ Teruo Noguchi ${ }^{1,2}$, Satoshi Yasuda ${ }^{1,2}$
}

${ }^{1}$ Department of Cardiovascular Medicine, National Cerebral \& Cardiovascular Center, Osaka, Japan; ${ }^{2}$ Department of Advanced Cardiovascular Medicine, Graduate School of Medical Sciences, Kumamoto University, Kumamoto, Japan; ${ }^{3}$ Department of Preventative Cardiology, National Cerebral \& Cardiovascular Center, Osaka, Japan; ${ }^{4}$ Division of Internal Medicine, Okinawa Prefectural Yaeyama Hospital, Okinawa, Japan; ${ }^{5}$ Department of Cardiology, Miyazaki Medical Association Hospital, Miyazaki, Japan; ${ }^{6}$ Department of Cardiovascular Medicine, Dokkyo Medical University Hospital, Tochigi, Japan; ${ }^{7}$ Department of Cardiovascular Medicine Graduate School of Medical Sciences, Kumamoto University, Kumamoto, Japan

Contributions: (I) Conception and design: K Murai, Y Kataoka; (II) Administrative support: S Yasuda; (III) Provision of study materials or patients: Y Kataoka; (IV) Collection and assembly of data: K Murai, Y Kataoka, S Kitahara, T Iwai, H Nakamura, H Hosoda; (V) Data analysis and interpretation: K Murai, Y Kataoka, Y Nakaoku, K Nishimura, S Yasuda; (VI) Manuscript writing: All authors; (VII) Final approval of manuscript: All authors.

Correspondence to: Yu Kataoka, MD, PhD, FAHA, FESC, FACC. Department of Cardiovascular Medicine, National Cerebral \& Cardiovascular Center, 6-1, Kishibe-shimmachi, Suita, Osaka, 564-8565, Japan. Email: yu.kataoka@ncvc.go.jp.

Background: Vulnerable plaque features including lipidic plaque have been shown to affect fractional flow reserve (FFR). Given that formation and propagation of lipid plaque is accompanied by endothelial dysfunction which impairs vascular tone, the degree of lipidic burden may affect vasoreactivity during hyperemia, potentially leading to reduced FFR. Our aim is to elucidate the relationship of the extent of lipidic plaque burden with coronary physiological vasoreactivity measure.

Methods: We analyzed 89 subjects requeuing PCI due to angiographically intermediate coronary stenosis with FFR $\leq 0.80$. Near-infrared spectroscopy (NIRS) and intravascular ultrasound were used to evaluate lipid-core burden index (LCBI) and atheroma volume at both target lesion (maxLCBI ${ }_{4 m m}$; maximum value of LCBI within any $4 \mathrm{~mm}$ segments) and entire target vessel $\left(\mathrm{LCBI}_{\text {vessel }}\right.$ LCBI within entire vessel). In addition to FFR, delta-FFR was measured by difference of distal coronary artery pressure/aortic pressure $(\mathrm{Pd} / \mathrm{Pa})$ between baseline and hyperemic state.

Results: The averaged FFR and delta-FFR was 0.74 (0.69-0.77), and $0.17 \pm 0.05$, respectively. On target lesion-based analysis, maxLCBI ${ }_{4 \mathrm{~mm}}$ was negatively correlated to FFR $(\rho=-0.213, \mathrm{P}=0.040)$, and it was positively correlated to delta-FFR $(\rho=0.313, \mathrm{P}=0.002)$. Furthermore, target vessel-based analysis demonstrated similar relationship of $\mathrm{LCBI}_{\text {vessel }}$ with FFR $(\rho=-0.302, \mathrm{P}=0.003)$ and delta-FFR $(\rho=0.369$, $\mathrm{P}<0.001)$. Even after adjusting clinical characteristics and lesion/vessel features, delta-FFR (by 0.10 increase) was independently associated with $\operatorname{maxLCBI}_{4 \mathrm{~mm}}(\beta=57.2, \mathrm{P}=0.027)$ and $\mathrm{LCBI}_{\text {vessel }}(\beta=24.8, \mathrm{P}=0.007)$ by mixed linear model analyses.

Conclusions: A greater amount of lipidic plaque burden at not only "target lesion" alone but "entire target vessel" was associated with a greater delta-FFR. The accumulation of lipidic plaque materials at both local site and entire vessel may impair hyperemia-induced vasoreactivity, which causes a reduced FFR. 
Keywords: Fractional flow reserve (FFR); near-infrared spectroscopy (NIRS); intravascular ultrasound; coronary artery disease

Submitted Dec 19, 2020. Accepted for publication Feb 15, 2021.

doi: $10.21037 / \mathrm{cdt}-20-1024$

View this article at: http://dx.doi.org/10.21037/cdt-20-1024

\section{Introduction}

Fractional flow reserve (FFR) has become the current gold standard to evaluate myocardial perfusion $(1,2)$. Recent studies reported that, in addition to anatomical characteristics, vulnerability of coronary lesions associated with $\operatorname{FFR}(3,4)$. Given an increased plaque vulnerability as a potential substrate causing future coronary events, this finding could account in part for the ability of FFR to predict subsequent cardiac outcome (5). However, its detailed mechanism remains to be fully determined yet.

The proposed mechanisms behind this observation is that vulnerable plaques could cause functional stenosis under hyperemic agents which dilate not only coronary resistance vessels but also epicardial coronary arteries (6). This is because vulnerable features including lipidic burden is triggered by endothelial dysfunction (7) affecting vasodilatory property at lesions (8). However, given the diffusely propagation of lipidic plaque accumulation within epicardial arteries (9), vasodilatory property at entire vessel may influence coronary physiology.

Delta-FFR, a change in $\mathrm{Pd} / \mathrm{Pa}$ (distal coronary artery pressure/aortic pressure) from baseline to hyperemic status is a clinical measure to evaluate the degree of vascular response to vasodilatory agent $(10,11)$. In addition, an intravascular near-infrared spectroscopy (NIRS) imaging enables to quantitatively visualize lipidic materials within vessel wall $(12,13)$. Therefore, we investigated the association of delta-FFR with NIRSderived lipid plaque measure in patients with stable CAD. We present the following study in accordance with the MDRA reporting checklist (available at: http://dx.doi. org/10.21037/cdt-20-1024).

\section{Methods}

The study protocol conforms to the ethical guidelines of the Declaration of Helsinki (as revised in 2013) and this study protocol was approved by the institutional ethics committee of National Cerebral and Cardiovascular Center (research project number: M30-084-2). Informed consent was not obtained in each subject due to the observational analysis of hospitalized patients.

\section{Study subjects}

This study is a single-center retrospective registry which consecutively enrolled any patients with intermediate coronary artery stenosis (FFR $\leq 0.80$ ) who underwent NIRS/intravascular ultrasound (IVUS) imaging prior to percutaneous coronary intervention (PCI). Intermediate stenosis was defined as a lesion with its percent diameter stenosis (\%DS) between $25 \%$ and $75 \%$ on quantitative coronary angiography (QAngio ${ }^{\circledR} \mathrm{XA}$, Medis, Leiden, the Netherlands). In patients with stable coronary artery disease (CAD) receiving PCI at National Cerebral and Cardiovascular Center from January $1^{\text {st }}, 2017$ to August $31^{\text {st }}, 2019,214$ patients had intermediate stenosis according to significant FFR value $(\leq 0.80)$ (Figure 1). Of these, NIRS/ IVUS imaging was used in 124 patients for the guidance of PCI procedure. We excluded the following patients from the current analysis; subjects who had a history of PCI within the target vessel $(n=11)$ or target vessel-related myocardial infarction $(n=8)$, those with coronary artery bypass graft $(n=4)$, those who required pre-dilatation before NIRS/IVUS imaging due to severe stenosis $(\mathrm{n}=13)$, and subjects with insufficient image quality $(\mathrm{n}=1)$ or missing delta-FFR values $(\mathrm{n}=3)$. As a consequence, the remaining 89 patients (94 vessels) were included into the current analysis (Figure 1).

\section{FFR measurements}

FFR measurement was performed to evaluate myocardial ischemia prior to PCI as previously described (14). In patients who required staged PCI, FFR measurements were performed prior to the initial PCI procedure. Briefly, after 5 or 6 French guide catheter $(n=84 / 5)$ was cannulated into the coronary artery, pressure-monitoring guidewire (PressureWire ${ }^{\mathrm{TM}}$ Cetus $^{\mathrm{TM}}$ or Aeris ${ }^{\mathrm{TM}}$, Abbot, 


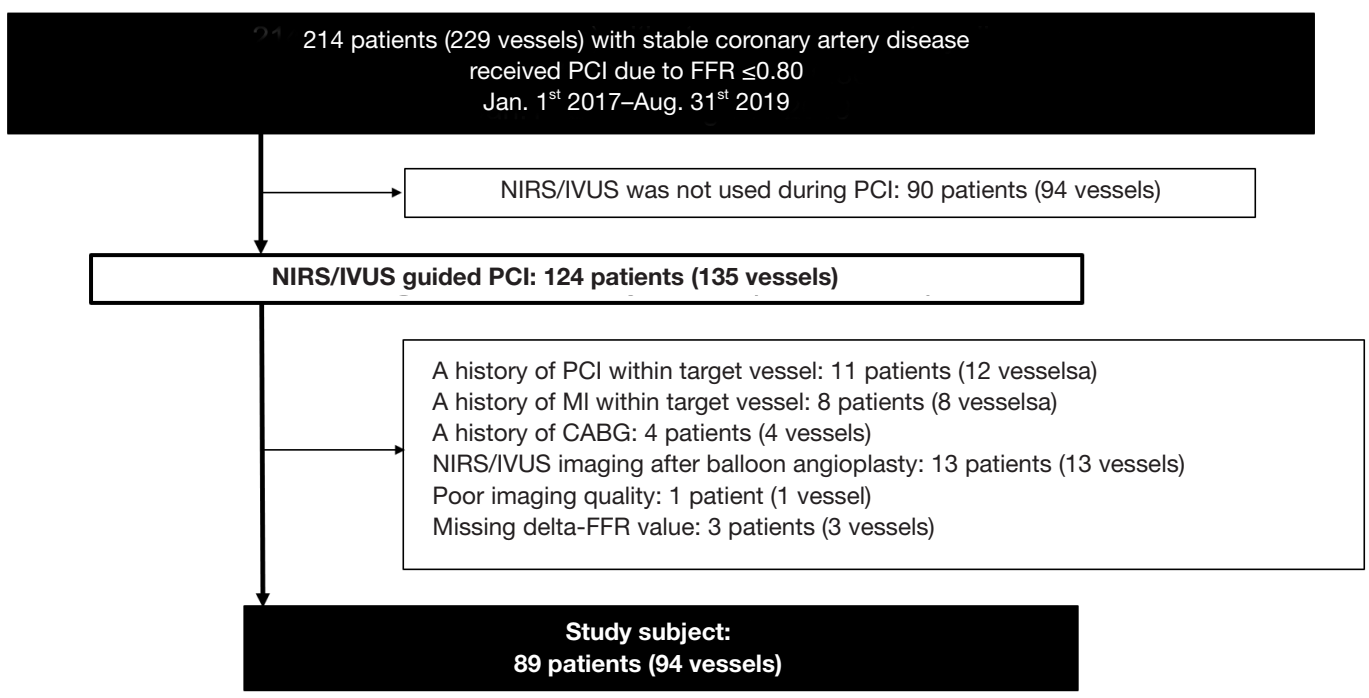

Figure 1 Patients' disposition. CABG, coronary artery bypass grafting; FFR, fractional flow reserve; IVUS, intravascular ultrasound; MI, myocardial infarction; NIRS, near-infrared spectroscopy; PCI, percutaneous coronary intervention.

Abbott Park, IL, USA; Optowire ${ }^{\circledR}$, ZEON MEDICAL, Tokyo, Japan) was advanced to the most distal site of the target vessel for PCI. Following the measurement of $\mathrm{Pd} / \mathrm{Pa}$ at rest, hyperemia was induced by administration of intravenous adenosine 5'-triphosphate (ATP) at the rate of $180 \mathrm{ug} / \mathrm{kg} / \mathrm{min}$. If the use of ATP was inadequate, intracoronary nicorandil with $2 \mathrm{mg}$ was alternatively used (15). After hyperemic state was obtained, $\mathrm{Pd} / \mathrm{Pa}$ was measured again $(=$ FFR). Additionally, delta-FFR was calculated by using the following formula:

$$
\text { delta-FFR }=\mathrm{Pd} / \mathrm{Pa} \text { at rest }-\mathrm{Pd} / \mathrm{Pa} \text { at hyperemia } .
$$

\section{NIRS/IVUS imaging acquisition}

The entire target vessel requiring PCI was evaluated by NIRS/IVUS imaging after FFR measurement. The median interval between FFR measurement and NIRS/ IVUS imaging was 35 days. In detail, after intracoronary administration of nitroglycerin $(100-300 \mu \mathrm{g})$, the imaging catheter (TVC Insight ${ }^{\mathrm{TM}}$ or Dualpro ${ }^{\mathrm{TM}}$, Infraredx, Bedford, MA, USA) was automatically pull backed from the most distal site of the target artery at a speed of $0.5 \mathrm{~mm} / \mathrm{sec}$ and $960 \mathrm{rpm}$ (TVC Insight ${ }^{\mathrm{TM}}$ ) or $2.0 \mathrm{~mm} / \mathrm{sec}$ and $1,800 \mathrm{rpm}$ (Dualpro $^{\mathrm{TM}}$ ) (12) The raw IVUS data was transferred to commercially available software, QIvus ${ }^{\circledR}$ (Medis, Leiden, the Netherlands) for quantitative analysis of IVUS images. With regard to NIRS data, Makoto ${ }^{\circledR}$ system (Infraredx,
Bedford, MA, USA) was used to analyze obtained chemogram data. Both analyses were conducted by persons who were blinded to the clinical characteristics of the patients (KM and $\mathrm{YK})$.

\section{Quantitative analysis of IVUS and NIRS images}

Quantitative analysis of IVUS and NIRS measures was conducted at both target lesion ("lesion-based analysis") and entire target vessel ("vessel-based analysis"), respectively. Target lesion was defined as the lesion where PCI was performed. If multiple significant stenosis existed, the most stenotic lesion was selected for the lesion-based analysis. The entire target vessel was defined as the segment from the ostium of the target artery to its most distal site where imaging catheter was able to be delivered.

IVUS analysis was conducted at each 1-mm interval cross-sectional image. The leading edges of the lumen and external elastic membrane (EEM) were traced by manual planimetry. Plaque area was defined as the area occupied between these leading edges $(16,17)$. Calcification was also analyzed at each image. Calcification grade was defined according to the arc of calcification; $0=$ no calcium, $1=$ calcification $\operatorname{arc}<90^{\circ}, 2=$ calcification $\operatorname{arc} \leq 90^{\circ}$ but $<180^{\circ}$; $3=$ calcification $\operatorname{arc} \leq 180^{\circ}$ but $\angle 270^{\circ}, 4=$ calcification $\operatorname{arc}$ $\leq 270^{\circ}(18)$. With regard to NIRS analysis, lipid-core burden index (LCBI) was automatically calculated as the number of yellow pixels within region of interest (ROI), divided by the 
total pixel quantity within the corresponding ROI (19).

The current study conducted these imaging analyses at target lesion and vessel, respectively.

\section{Target lesion-based analysis}

Minimum lumen area (MLA), maximum plaque area and maximum value of LCBI within any $4 \mathrm{~mm}$ segment $\left(\operatorname{maxLCBI}{ }_{4 \mathrm{~mm}}\right)$ were analyzed through IVUS and NIRS images at target lesion (13). The maximum calcification grade at target lesion was used for this analysis.

\section{Target vessel-based analysis}

IVUS and NIRS measures at entire vessel were analyzed. The total atheroma volume (TAV) was calculated by summation of the plaque area calculated for each measured image and subsequently normalized to account for difference in segment length between subjects $(16,17)$ :

$$
\begin{aligned}
T A V_{\text {Normalized }} & =\frac{\sum\left(\text { EEM }_{\text {area }}-\text { Lumen }_{\text {area }}\right)}{\text { Number of Images in Pullback }} \\
& \times \text { Median number of images in cohort }
\end{aligned}
$$

The percent atheroma volume (PAV) was calculated as the proportion of vessel wall volume occupied by atherosclerotic plaque $(16,17)$ :

$$
P A V=\frac{\sum\left(E E M_{\text {area }}-\text { Lumen }_{\text {area }}\right)}{\sum E E M_{\text {area }}} \times 100
$$

Volumes occupied by the lumen and EEM were similarly calculated by summation of their respective areas in each measured image and subsequently normalized to account for differences in segment length between subjects. The maximum calcification grade throughout the target vessel was used for this analysis. With regard to NIRS analysis, LCBI within the entire target vessel $\left(\mathrm{LCBI}_{\text {vessel }}\right)$ was measured for vessel-based analysis.

\section{Statistical analysis}

Continuous values with normal distribution were expressed as mean \pm SD. Normality of distribution was tested with the Shapiro-Wilk test. Variables with non-normal distribution were expressed with median (interquartile range). Categorical data were expressed with $\mathrm{n}(\%)$. Comparisons of continuous variables with normal distribution were tested by 1-way ANOVA with Tukey's multiple comparison, and variables with non-normal distribution were tested by Kruskal-Wallis test with Dunn's nonparametric comparison with Bonferroni adjustment. $\mathrm{LCBI}_{\text {vessel }}$ was examined using analysis of covariance (ANCOVA), with $\mathrm{Pd} / \mathrm{Pa}$ at rest and $\mathrm{PAV}$ as a covariate and grouping variable (tertile of deltaFFR) as a factor. Similarly, maxLCBI ${ }_{4 \mathrm{~mm}}$ was examined with $\mathrm{Pd} / \mathrm{Pa}$ at rest and MLA as a covariate. Categorical variables were analyzed by $\chi^{2}$ test. Correlation analysis was performed with Spearman's correlation analysis.

We fit multivariable linear mixed-effects models for lipidic burden ( $\max \mathrm{LCBI}_{4 \mathrm{~mm}}, \mathrm{LCBI}_{\text {vessel }}$ ) with subjectspecific random intercepts to evaluate the effect of clinical characteristics, angiographical, IVUS, and physiological measures per lesion and vessel. All statistical tests were 2 sided, and $\mathrm{P}<0.05$ was regarded as statistically significant. These analyses were performed with SPSS software version 21 (IBM Corporation, Armonk, NY, USA) and STATA version 15 (StataCorp LP, College Station, TX, USA).

\section{Results}

\section{Clinical characteristics of study subjects}

Clinical demographics of study population are summarized in Table 1. Patients had a mean age of 72 years, $85 \%$ were male, and they had a high prevalence of risk factors (hypertension: 75\%, dyslipidemia: 84\%, type 2 diabetes mellitus: $42 \%$ ). Almost a quarter of patients exhibited effort angina pectoris and the remaining patients were diagnosed as silent myocardial ischemia. The majority of study subjects already received aspirin (97\%), $\mathrm{P} 2 \mathrm{Y}_{12}$ inhibitor (96\%) and statin $(97 \%)$ at the index of the procedure (Table 1).

\section{Physiological measures}

Table 2 summarizes physiological measures in the current study subjects. Over $80 \%$ of analyzed target lesions was located within left anterior descending artery, followed by left circumflex artery (11\%) and right coronary artery (7\%). ATP was used for FFR measurement in most of vessels. The averaged FFR and delta-FFR were $0.74(0.69-0.77)$, and $0.17 \pm 0.05$, respectively. $56 \%$ of vessels exhibited FFR $<0.75$ (Table 2). 
Table 1 Baseline characteristics $(\mathrm{n}=89)$

\begin{tabular}{|c|c|}
\hline Characteristics & Value \\
\hline Age, years & 72 [62-78] \\
\hline Male, n (\%) & $76(85)$ \\
\hline Body mass index, $\mathrm{kg} / \mathrm{m}^{2}$ & $24.4 \pm 3.2$ \\
\hline Effort angina pectoris, $\mathrm{n}(\%)$ & $23(26)$ \\
\hline Silent myocardial ischemia, $\mathrm{n}(\%)$ & $66(74)$ \\
\hline \multicolumn{2}{|l|}{ Coronary risk factor, n (\%) } \\
\hline Hypertension & $67(75)$ \\
\hline Dyslipidemia & $75(84)$ \\
\hline Diabetes mellitus & $37(42)$ \\
\hline Current smoking & $14(16)$ \\
\hline Family history of coronary artery disease & $17(19)$ \\
\hline Previous myocardial infarction & $16(17)$ \\
\hline \multicolumn{2}{|l|}{ Laboratory data } \\
\hline eGFR, mL/min/1.73 m² & $62.9 \pm 18.2$ \\
\hline LDL-cholesterol, mg/dL & $81.3 \pm 25.1$ \\
\hline HDL-cholesterol, mg/dL & $47.4 \pm 11.7$ \\
\hline Hemoglobin A1c, \% & $6.0(5.7-6.8)$ \\
\hline \multicolumn{2}{|l|}{ Medication, n (\%) } \\
\hline Aspirin & $86(97)$ \\
\hline $\mathrm{P} \mathrm{Y}_{12}$ inhibitor & $85(96)$ \\
\hline Statin & $86(97)$ \\
\hline Ezetimibe & $10(11)$ \\
\hline Beta blocker & $58(65)$ \\
\hline ACE-I/ARB & $57(59)$ \\
\hline
\end{tabular}

ACE-I, angiotensin-converting-enzyme inhibitor; ARB, angiotensin II receptor blocker; eGFR, estimated glomerular filtration rate; HDL, high-density lipoproteins; LDL, low-density lipoproteins.

\section{NIRS/IVUS measures}

At target lesions, their MLA, maximum percent plaque area and maxLCBI $_{4 \mathrm{~mm}}$ were $1.7(1.5-2.2) \mathrm{mm}^{2}, 84.1 \%$ (79.4-87.3\%) and 398.7 \pm 172.3 , respectively (Table 3). In addition, $45 \%$ of target lesions exhibited their maximum calcification grade $\leq 3$. Under the imaging of entire vessels with its length of $79.2 \pm 17.9 \mathrm{~mm}$, normalized TAV, PAV and the corresponding NIRS-derived LCBI $_{\text {vessel }}$ at target vessel were $485.7(409.5-643.9) \mathrm{mm}^{3}, 50.15 \% \pm 7.4 \%$ and 73
Table 2 FFR demographics

\begin{tabular}{lc}
\hline Demographics & Value \\
\hline Location of target lesion & $77(82)$ \\
LAD, $\mathrm{n}(\%)$ & $54(57)$ \\
Proximal segment of LAD, $\mathrm{n}(\%)$ & $10(11)$ \\
LCX, $\mathrm{n}(\%)$ & $2(2)$ \\
Proximal segment of LCX, $\mathrm{n}(\%)$ & $7(7)$ \\
RCA, n (\%) & $3(3)$ \\
Proximal segment of RCA, $\mathrm{n}(\%)$ & \\
FFR measures & $91(97)$ \\
ATP use, $\mathrm{n}(\%)$ & $3(3)$ \\
Nicorandil use, $\mathrm{n}(\%)$ & $0.90(0.87-0.92)$ \\
Pd/Pa at rest & $0.74(0.69-0.77)$ \\
FFR & $0.17 \pm 0.05$ \\
Delta-FFR
\end{tabular}

ATP, adenosine 5'-triphosphate; FFR, fractional flow reserve; LAD, left anterior descending artery; LCX, left circumflex artery; $\mathrm{Pd} / \mathrm{Pa}$, distal coronary artery pressure/aortic pressure; RCA, right coronary artery.

[31-111], respectively (Table 3). Maximum calcification grade $\leq 3$ was observed at $48 \%$ of target vessels.

\section{Association of LCBI with FFR and delta-FFR}

\section{Target lesion-based analysis}

The relationship of maxLCBI $\mathrm{Cmm}_{4 \mathrm{~m}}$ at target lesions with FFR and delta-FFR was illustrated by scatterplots as shown in Figure $2 A, B$. FFR was significantly and negatively correlated to maxLCBI ${ }_{4 \mathrm{~mm}}(\rho=-0.213, \mathrm{P}=0.040$, Figure $2 A)$. Furthermore, delta-FFR was significantly and positively correlated to $\operatorname{maxLCBI}_{4 \mathrm{~mm}}(\rho=0.313 \mathrm{P}=0.002$, Figure $2 B)$.

\section{Target vessel-based Analysis}

The aforementioned relationship was similarly observed by vessel-based analysis. Both FFR and delta-FFR were significantly associated with $\mathrm{LCBI}_{\text {vessel }}(\rho=-0.302, \mathrm{P}=0.003$ and $\rho=0.369, \mathrm{P}<0.001$ ) (Figure $2 C, D$ ).

\section{Atheroma characteristics and delta-FFR}

Further analysis was conducted to elucidate plaque features associated with delta-FFR. Imaged vessels were stratified according to tertile of delta-FFR (delta-FFR $<0.15,0.15$ 
Table 3 Angiographic and NIRS/IVUS Demographics

\begin{tabular}{|c|c|}
\hline & Value \\
\hline \multicolumn{2}{|l|}{ Target lesion-based data (94 lesions) } \\
\hline \multicolumn{2}{|l|}{ QCA analysis } \\
\hline$\% D S, \%$ & $53.7 \pm 11.1$ \\
\hline Lesion length, mm & $12.0(9.0-17.3)$ \\
\hline \multicolumn{2}{|l|}{ IVUS measures } \\
\hline Minimum lumen area, $\mathrm{mm}^{2}$ & $1.7(1.5-2.2)$ \\
\hline Maximum percent plaque area, \% & $84.1(79.4-87.3)$ \\
\hline \multicolumn{2}{|l|}{ Maximum calcium grade, $n(\%)$} \\
\hline 0 & $6(6)$ \\
\hline 1,2 & $46(49)$ \\
\hline 3,4 & $42(45)$ \\
\hline \multicolumn{2}{|l|}{ NIRS measure } \\
\hline $\operatorname{maxLCBI}{ }_{4 \mathrm{~mm}}$ & $398.7 \pm 172.3$ \\
\hline \multicolumn{2}{|l|}{ Target vessel-based data (94 vessels) } \\
\hline \multicolumn{2}{|l|}{ IVUS measures } \\
\hline \multicolumn{2}{|l|}{ Culprit vessel measures } \\
\hline Imaged length, $\mathrm{mm}$ & $79.8 \pm 17.9$ \\
\hline Vessel volume, $\mathrm{mm}^{3}$ & $965.9(780.4-1,225.9)$ \\
\hline Lumen volume, $\mathrm{mm}^{3}$ & $487.7(381.5-626.9)$ \\
\hline Normalized TAV, $\mathrm{mm}^{3}$ & $485.7(409.5-643.9)$ \\
\hline PAV, \% & $50.1 \pm 7.4$ \\
\hline \multicolumn{2}{|l|}{ Maximum calcium grade, n (\%) } \\
\hline 0 & $2(2)$ \\
\hline 1,2 & $47(50)$ \\
\hline 3,4 & $45(48)$ \\
\hline \multicolumn{2}{|l|}{ NIRS measure } \\
\hline LCBI $_{\text {vessel }}$ & $73[31-111]$ \\
\hline
\end{tabular}

IVUS, intravascular ultrasound; LCBI, lipid core burden index; NIRS, near-infrared spectroscopy; \%DS, percent diameter stenosis; QCA, quantitative coronary angiography; TAV, total atheroma volume; PAV, percent atheroma volume.

0.18 , and $>0.18$ ) (Table 4). Vessels with a larger delta-FFR were more likely to exhibit a higher $\mathrm{Pd} / \mathrm{Pa}$ at rest and a reduced FFR. On NIRS/IVUS imaging, while there were no significant differences in measures of \% DS, atheroma burden and maximum calcification grade across delta-FFR values, $\operatorname{maxLCBI}_{4 \mathrm{~mm}}(\mathrm{P}=0.01)$ and higher $\mathrm{LCBI}_{\text {vessel }}(\mathrm{P}=0.02)$ were observed pertinent to an increase in delta-FFR. These observations were still consistent even after adjusting atheroma volume and $\mathrm{Pd} / \mathrm{Pa}$ at rest (Table 4).

Mixed linear model analyses were performed to identify the relationship between delta-FFR and lipidic burden (Table 5). On target-lesion based multivariate analysis adjusting clinical characteristics and lesion/vessel features, delta-FFR $(\mathrm{P}=0.020), \% \mathrm{DS}(\mathrm{P}=0.013)$ and $\mathrm{PAV}(\mathrm{P}<0.001)$ were significant predictors of maxLCBI ${ }_{4 \mathrm{~mm}}$ (Table 5). Targetvessel based multivariate analysis also revealed that these measures predicted $\mathrm{LCBI}_{\text {vessel }}$ (delta-FFR: $\mathrm{P}=0.002, \% \mathrm{DS}$ : $\mathrm{P}=0.015$, PAV: $\mathrm{P}<0.001$ ) (Table 5). Figure 3 illustrates two representative cases.

\section{Discussion}

Recent studies have reported a potential role of plaque vulnerability in reduced FFR $(4,20)$. However, the mechanism behind this observation remains to be fully determined yet. In the current study, NIRS-derived LCBI at both target lesions and entire vessels was associated with not only FFR but delta-FFR. Even after adjusting atheroma volume and angiographic features, these lipidic plaque measure independently associated with delta-FFR. Our findings suggest a pathophysiological role of lipidic plaque component within entire vessel in determining FFR.

Current observation indicates the association of plaque instability with coronary physiological abnormality. In our analysis, LCBI affected delta-FFR, which reflects vasoreactivity under vasodilatory agent. In general, lipid plaque is formulated through the influx of atherogenic inflammatory cytokines and oxidative stress, causing endothelial dysfunction (21). This mechanism is supported by another study which demonstrated severer endothelial dysfunction at target lesion harboring a great amount of necrotic core materials $(20,22)$. Since the endothelium regulates vascular tone (8), lipidic atheroma with endothelial dysfunction may cause insufficient vascular response during hyperemic status, potentially leading to a greater deltaFFR. Our findings underscore the amount of lipid plaque component as an additional determinant of coronary physiology.

Another novel observation is that delta-FFR is associated with LCBI at not only "target lesion" alone but "entire target vessel". Given that accumulation of lipid plaque does not occur locally but normally propagate at coronary tree (9), this nature of atherosclerosis could induce the diffusely attenuated vascular response at entire vessel. In 
A

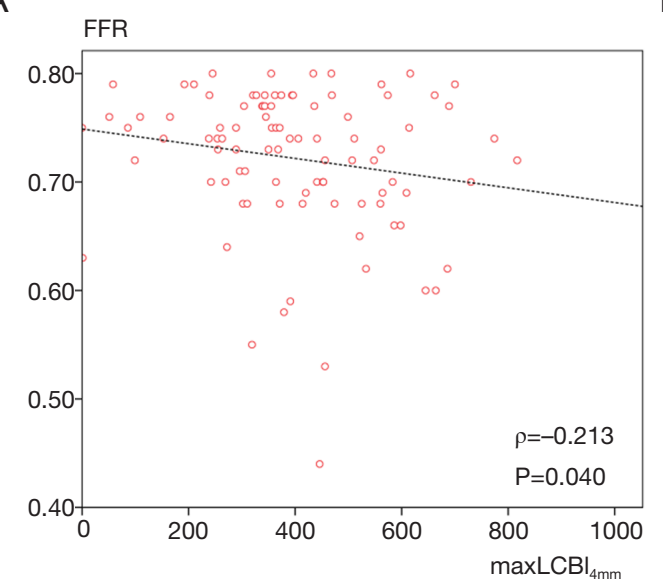

C

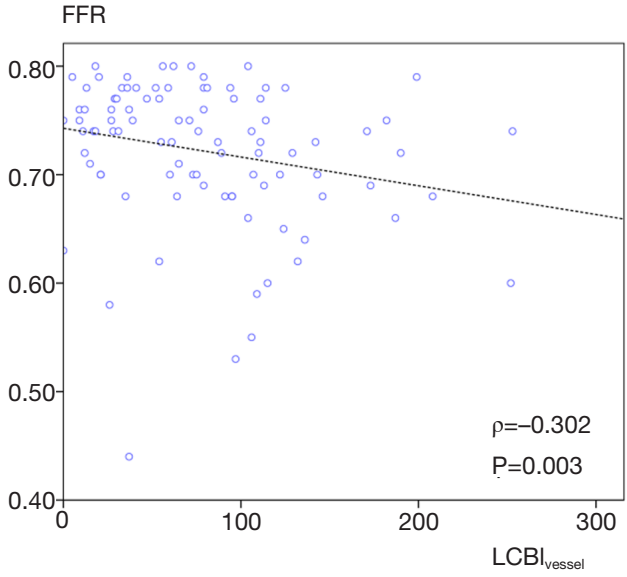

B

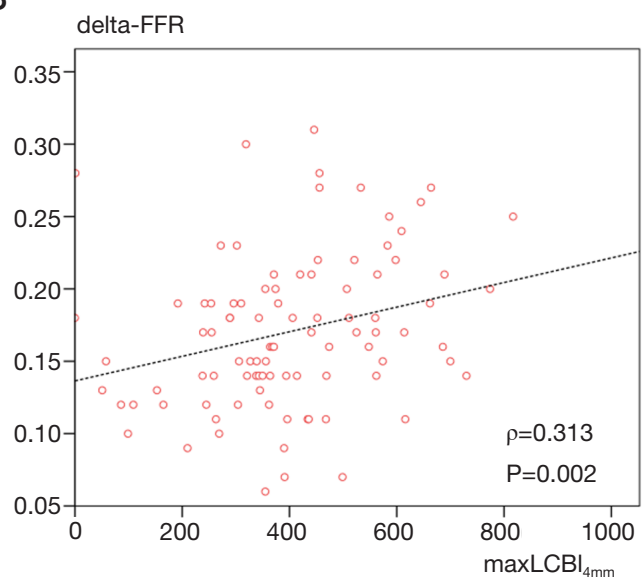

D

delta-FFR

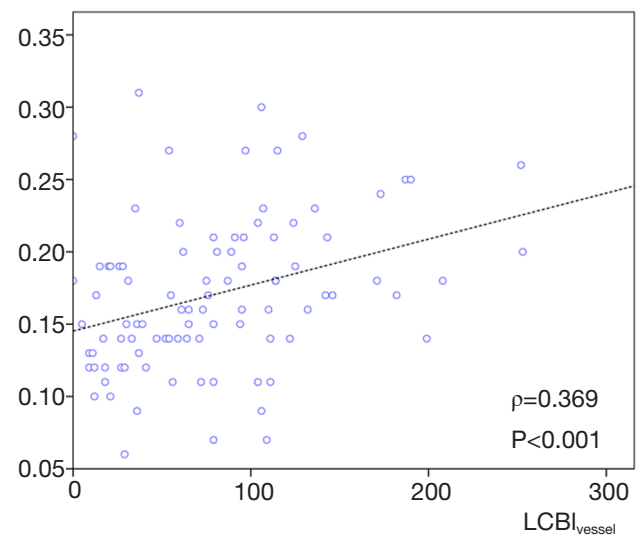

Figure 2 Physiological measures and LCBI at target lesion. (A) Correlation between FFR and maxLCBI ${ }_{4 m m}$; (B) Correlation between delta-

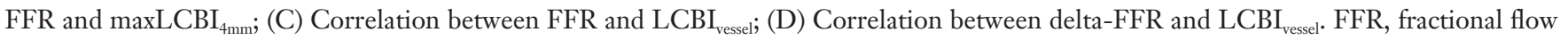
reserve; LCBI, lipid core burden index.

other studies, diffuse coronary atherosclerosis has been shown to cause abnormal epicardial coronary artery resistance along with reduced myocardial blood flow $(23,24)$. These findings highlight that propagation of lipidic plaques within whole coronary artery may play an important role in negatively influencing coronary physiology.

In the current analysis, the correlation coefficient between physiological measures and NIRS-derived measure is low, although it is statistically significant. One of the potential reasons behind this observation is that coronary physiological measures could be affected by other multiple factors such as stenosis severity, plaque burden, lesion length (25). Further investigation will be warranted to evaluate which factor is more dominant one associated with delta-FFR.
Coronary physiological evaluation may enable to predict the presence of lipid-rich atheroma which requiring intensive anti-atherosclerotic management. In our analysis, CAD subjects with a larger delta-FFR more likely exhibited an elevated LCBI. Given an increased risk for subsequent coronary events in patients with a higher LCBI (26), evaluation of not only FFR but also delta-FFR may help to identify stable CAD patients who warrant intensified optimal medical therapy including potent lipid-lowering agent. Future dedicated studies will be required to elucidate whether (I) delta-FFR could predict future cardiovascular events and (II) intensification of anti-atherosclerotic medical therapies based on delta-FFR could improve cardiovascular outcomes in patients with CAD.

The current study has several limitations. Firstly, this 
Table 4 Angiographic and NIRS/IVUS features, and delta-FFR

\begin{tabular}{|c|c|c|c|c|}
\hline & Delta-FFR <0.15 $(n=34)$ & $0.15 \leq$ delta-FFR $\leq 0.18(n=27)$ & $0.18<$ delta-FFR $(n=33)$ & $P$ value \\
\hline $\mathrm{Pd} / \mathrm{Pa}$ at rest & $0.89(0.85-0.91)$ & $0.91(0.88-0.93)$ & $0.91(0.87-0.94)$ & 0.02 \\
\hline FFR & $0.76(0.74-0.78)$ & $0.74(0.71-0.77)$ & $0.69(0.64-0.72)$ & $<0.001$ \\
\hline delta-FFR & $0.12 \pm 0.02$ & $0.17 \pm 0.01$ & $0.23 \pm 0.04$ & $<0.001$ \\
\hline LAD, n (\%) & $30(88)$ & $23(85)$ & $24(73)$ & 0.22 \\
\hline$\% D S$ at culprit lesion, $\%$ & $53.0 \pm 10.0$ & $53.5 \pm 10.5$ & $54.4 \pm 12.8$ & 0.88 \\
\hline Length of culprit lesion, mm & $13.0(9.8-17.3)$ & $13.0(11.0-18.0)$ & $11.0(8.0-17.0)$ & 0.14 \\
\hline \multicolumn{5}{|l|}{ IVUS analysis } \\
\hline MLA at culprit lesion, $\mathrm{mm}^{2}$ & $1.6(1.5-2.1)$ & $1.7(1.4-2.2)$ & $1.6(1.5-2.3)$ & 0.90 \\
\hline $\begin{array}{l}\text { Maximum calcification grade within } \\
\text { target lesion }\end{array}$ & $2.5[2-4]$ & $2[1-3]$ & $3[2-3]$ & 0.21 \\
\hline $\begin{array}{l}\text { Maximum calcification grade within } \\
\text { target vessel }\end{array}$ & $3[2-4]$ & $2[2-3]$ & $3[2-3]$ & 0.13 \\
\hline \multicolumn{5}{|l|}{ NIRS analysis } \\
\hline $\operatorname{maxLCBI}_{4 \mathrm{~mm}}$ & $336.1 \pm 151.0$ & $405.8 \pm 168.0$ & $457.3 \pm 179.1$ & 0.01 \\
\hline Adjusted maxLCBI ${ }_{4 \mathrm{~mm}}{ }^{*}($ mean $\pm \mathrm{SE})$ & $330.5 \pm 77.3$ & $412.0 \pm 84.6$ & $457.8 \pm 76.7$ & 0.01 \\
\hline
\end{tabular}

Values are mean $\pm \mathrm{SD}$, \% or median (interquartile range). FFR, fractional flow reserve; IVUS, intravascular ultrasound; LAD, left anterior descending artery; LCBI, lipid core burden index; MLA, minimum lumen area; NIRS, near-infrared spectroscopy; PAV, percent atheroma volume; \%DS, percent diameter stenosis; Pd/Pa, distal coronary artery pressure/aortic pressure; SD, standard deviation; TAV, total atheroma volume. \#adjusted by $\mathrm{Pd} / \mathrm{Pa}$ at rest and MLA; *adjusted by $\mathrm{Pd} / \mathrm{Pa}$ at rest and PAV.

study was a single-center retrospective observational study. Secondly, FFR measurement and the use of NIRS/IVUS imaging were made according to the discretion of the individual PCI operator. This may cause selection bias. Thirdly, since we did not include patients with FFR $>0.80$, whether the current findings can be applied to patients with FFR $>0.80$ requires further investigation. Fourthly, given that ATP or nicorandil was used to measure FFR, it is unknown if the current findings can be translated to FFR data measured by using papaverine or other vasodilatory agents.
In conclusion, a greater amount of lipidic plaque burden at not only "target lesion" alone but "entire target vessel" was associated with a reduced FFR in CAD subjects with FFR $\leq 0.80$. Of note, these lipidic features predicted a greater delta-FFR in patients with stable CAD. These findings indicate that the accumulation of lipidic plaque materials at both local site and entire vessel may impair hyperemia-induced vasoreactivity, which causes a reduced FFR.

\section{Acknowledgments}


Table 5 Univariate and multivariate linear mixed model analysis for LCBI

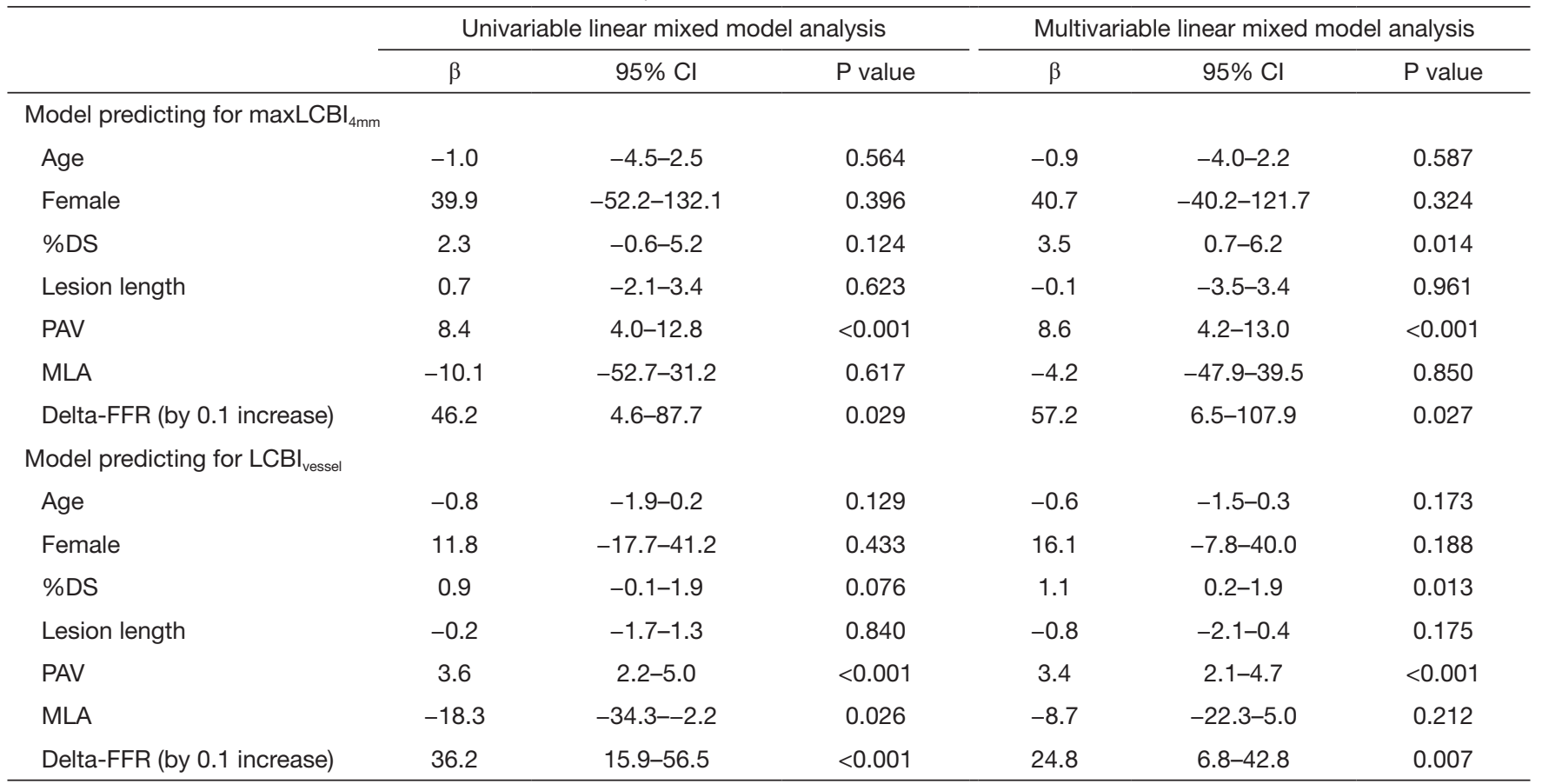

$\mathrm{CI}$, confidential interval; FFR, fractional flow reserve; LCBI, lipid core burden index; MLA, minimum lumen area; PAV, percent atheroma volume; \%DS, percent diameter stenosis.

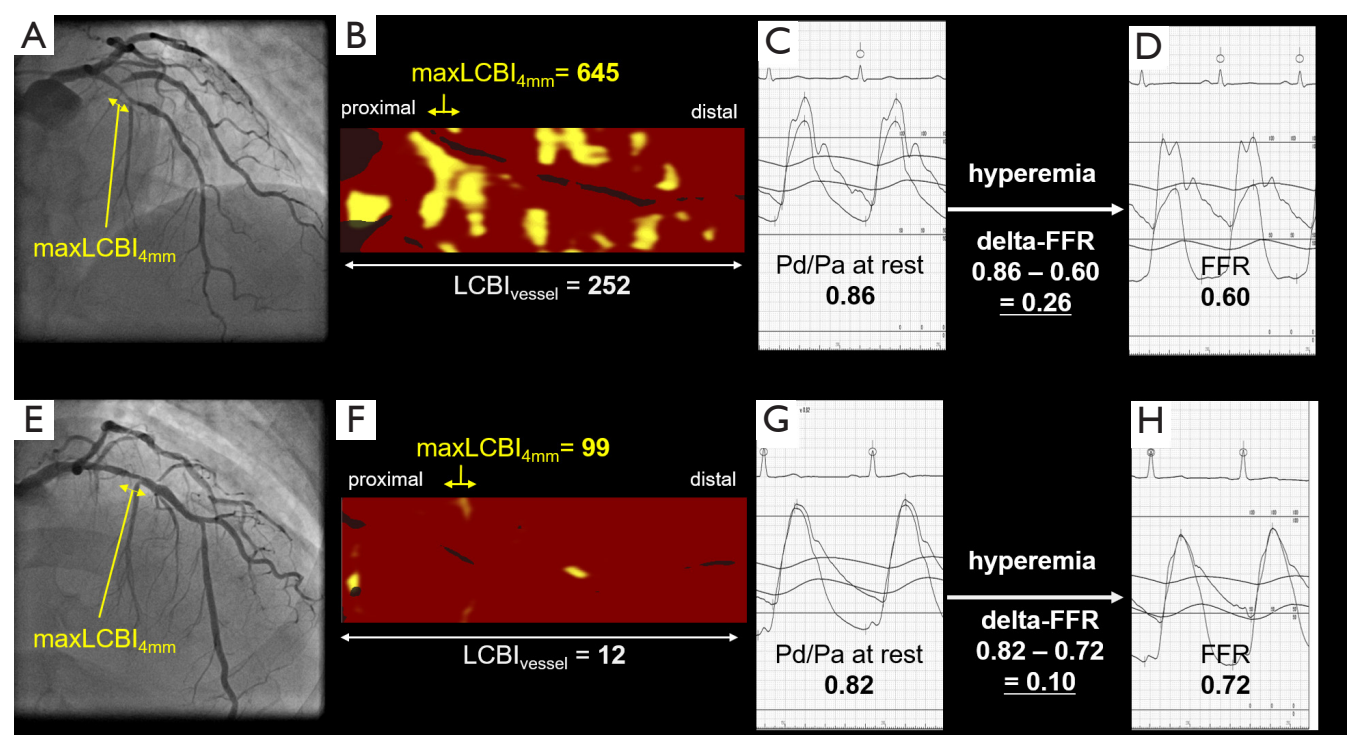

Figure 3 Representative cases. (A) A 58 years old woman received elective PCI for intermediate stenosis at the proximal segment of LAD. (B) NIRS imaging prior to PCI demonstrated extensive lipid accumulation, reflected by maxLCBI ${ }_{4 m m}$ and $\mathrm{LCBI}_{\text {vessel }}$ at 645 and 252 , respectively. (C and D) Distal coronary artery pressure/aortic pressure $(\mathrm{Pd} / \mathrm{Pa})$ substantially decreased from 0.86 to 0.60 during hyperemia, and its deltaFFR was 0.26. (E) A 75 years old man exhibited intermediate stenosis at the proximal segment of LAD. (F) On NIRS imaging, lower levels of $\operatorname{maxLCBI}{ }_{4 \mathrm{~mm}}(=99)$ and $\mathrm{LCBI}_{\text {vessel }}(=12)$ were observed. (G and $\mathrm{H}$ ) Reduction of corresponding Pd/Pa was identified from 0.82 to 0.72 under hyperemia. Its delta-FFR was 0.10 , which was smaller than the first case. FFR, fractional flow reserve; LAD, left anterior descending artery; LCBI, lipid core burden index; NIRS, near-infrared spectroscopy; PCI, percutaneous coronary intervention. 
We would like to acknowledge cardiology medical fellows and co-medical staffs for their support to conduct NIRS/ IVUS imaging and FFR measurements during PCI.

Funding: None.

\section{Footnote}

Reporting Checklist: The authors have completed the MDRA Checklist. Available at: http://dx.doi. org/10.21037/cdt-20-1024

Data Sharing Statement: Available at: http://dx.doi. org/10.21037/cdt-20-1024

Conflicts of Interest: All authors have completed the ICMJE uniform disclosure form (available at: http://dx.doi. org/10.21037/cdt-20-1024). Kota Murai has received honoraria from Abbot, ZEON MEDICAL. Yu Kataoka serves as an unpaid editorial board member of Cardiovascular Diagnosis and Therapy from Jul 2019 to Jun 2021, and has received research support from Nipro and Abbott, and honoraria from Nipro, Abbott, Kowa, Amgen, Sanofi, Astellas, Takeda and Daiichi-Sankyo. The other author has no conflicts of interest to declare.

Ethical Statement: The authors are accountable for all aspects of the work in ensuring that questions related to the accuracy or integrity of any part of the work are appropriately investigated and resolved. The study was conducted in accordance with the Declaration of Helsinki (as revised in 2013). The study was approved by the institutional ethics committee of National Cerebral and Cardiovascular Center (research project number: M30084-2) and individual consent was waived due to the observational analysis of hospitalized patients.

Open Access Statement: This is an Open Access article distributed in accordance with the Creative Commons Attribution-NonCommercial-NoDerivs 4.0 International License (CC BY-NC-ND 4.0), which permits the noncommercial replication and distribution of the article with the strict proviso that no changes or edits are made and the original work is properly cited (including links to both the formal publication through the relevant DOI and the license). See: https://creativecommons.org/licenses/by-nc-nd/4.0/.

\section{References}

1. Patel MR, Calhoon JH, Dehmer GJ, et al. ACC/AATS/ AHA/ASE/ASNC/SCAI/SCCT/STS 2017 Appropriate Use Criteria for Coronary Revascularization in Patients With Stable Ischemic Heart Disease. J Am Coll Cardiol 2017;69:2212-41.

2. Neumann FJ, Sousa-Uva M, Ahlsson A, et al. 2018 ESC/ EACTS Guidelines on myocardial revascularization. Eur Heart J 2019;40:87-165.

3. Park HB, Heo R, B OH, et al. Atherosclerotic plaque characteristics by $\mathrm{CT}$ angiography identify coronary lesions that cause ischemia: a direct comparison to fractional flow reserve. JACC Cardiovasc Imaging 2015;8:1-10.

4. Usui E, Yonetsu T, Kanaji Y, et al. Optical Coherence Tomography-Defined Plaque Vulnerability in Relation to Functional Stenosis Severity and Microvascular Dysfunction. JACC Cardiovasc Interv 2018;11:2058-68.

5. De Bruyne B, Pijls NH, Kalesan B, et al. Fractional flow reserve-guided PCI versus medical therapy in stable coronary disease. N Engl J Med 2012;367:991-1001.

6. Lupi A, Buffon A, Finocchiaro ML, et al. Mechanisms of adenosine-induced epicardial coronary artery dilatation. Eur Heart J 1997;18:614-7.

7. Otsuka F, Finn AV, Yazdani SK, et al. The importance of the endothelium in atherothrombosis and coronary stenting. Nat Rev Cardiol 2012;9:439-53.

8. Lerman A, Burnett JC, Jr. Intact and altered endothelium in regulation of vasomotion. Circulation 1992;86:III12-9.

9. Wykrzykowska JJ, Mintz GS, Garcia-Garcia HM, et al. Longitudinal distribution of plaque burden and necrotic core-rich plaques in nonculprit lesions of patients presenting with acute coronary syndromes. JACC Cardiovasc Imaging 2012;5:S10-8.

10. Kocaman SA, Sahinarslan A, Arslan U, et al. The delta fractional flow reserve can predict lesion severity and longterm prognosis. Atherosclerosis 2009;203:178-84.

11. Seo KW, Lim HS, Yoon MH, et al. The impact of microvascular resistance on the discordance between anatomical and functional evaluations of intermediate coronary disease. EuroIntervention 2017;13:e185-92.

12. Kataoka Y, Puri R, Andrews J, et al. In vivo visualization of lipid coronary atheroma with intravascular nearinfrared spectroscopy. Expert Rev Cardiovasc Ther 2017;15:775-85.

13. Madder RD, Goldstein JA, Madden SP, et al. Detection by Near-Infrared Spectroscopy of Large Lipid Core Plaques 
at Culprit Sites in Patients With Acute ST-Segment Elevation Myocardial Infarction. JACC: Cardiovascular Interventions 2013;6:838-46.

14. De Bruyne B, Pijls NH, Smith L, et al. Coronary thermodilution to assess flow reserve: experimental validation. Circulation 2001;104:2003-6.

15. Jang HJ, Koo BK, Lee HS, et al. Safety and efficacy of a novel hyperaemic agent, intracoronary nicorandil, for invasive physiological assessments in the cardiac catheterization laboratory. Eur Heart J 2013;34:2055-62.

16. Nicholls SJ, Tuzcu EM, Sipahi I, et al. Intravascular ultrasound in cardiovascular medicine. Circulation 2006;114:e55-9.

17. Nicholls SJ, Puri R, Anderson T, et al. Effect of Evolocumab on Progression of Coronary Disease in Statin-Treated Patients: The GLAGOV Randomized Clinical Trial. JAMA 2016;316:2373-84.

18. Puri R, Nicholls SJ, Shao $M$,et al. Impact of Statins on Serial Coronary Calcification During Atheroma Progression and Regression. J Am Coll Cardiol 2015;65:1273-82.

19. Gardner CM, Tan H, Hull EL, et al. Detection of lipid core coronary plaques in autopsy specimens with a novel catheter-based near-infrared spectroscopy system. JACC Cardiovasc Imaging 2008;1:638-48.

20. Puri R, Nicholls SJ, Brennan DM, et al. Coronary atheroma composition and its association with segmental endothelial dysfunction in non-ST segment elevation myocardial infarction: novel insights with radiofrequency

Cite this article as: Murai K, Kataoka Y, Nakaoku Y, Nishimura K, Kitahara S, Iwai T, Nakamura H, Hosoda H, Hirayama A, Matama H, Doi T, Nakashima T, Honda S, Fujino M, Nakao K, Yoneda S, Nishihira K, Kanaya T, Otsuka F, Asaumi Y, Tsujita K, Noguchi T, Yasuda S. The association between the extent of lipidic burden and delta-fractional flow reserve: analysis from coronary physiological and nearinfrared spectroscopic measures. Cardiovasc Diagn Ther 2021;11(2):362-372. doi: 10.21037/cdt-20-1024
(iMAP) intravascular ultrasonography. Int J Cardiovasc Imaging 2015;31:247-57.

21. Bonetti PO, Lerman LO, Lerman A. Endothelial dysfunction: a marker of atherosclerotic risk. Arterioscler Thromb Vasc Biol 2003;23:168-75.

22. Lavi S, Bae JH, Rihal CS, et al. Segmental coronary endothelial dysfunction in patients with minimal atherosclerosis is associated with necrotic core plaques. Heart 2009;95:1525-30.

23. Rizvi A, Hartaigh BO, Danad I, et al. Diffuse coronary artery disease among other atherosclerotic plaque characteristics by coronary computed tomography angiography for predicting coronary vessel-specific ischemia by fractional flow reserve. Atherosclerosis 2017;258:145-51.

24. De Bruyne B, Hersbach F, Pijls NH, et al. Abnormal epicardial coronary resistance in patients with diffuse atherosclerosis but "Normal" coronary angiography. Circulation 2001;104:2401-6.

25. Driessen RS, Stuijfzand WJ, Raijmakers PG, et al. Effect of Plaque Burden and Morphology on Myocardial Blood Flow and Fractional Flow Reserve. J Am Coll Cardiol 2018;71:499-509.

26. Waksman R, Di Mario C, Torguson R, et al. Identification of patients and plaques vulnerable to future coronary events with near-infrared spectroscopy intravascular ultrasound imaging: a prospective, cohort study. Lancet 2019;394:1629-37. 